\title{
On Performance of Energy Harvested Cooperative NOMA Under Imperfect CSI and Imperfect SIC
}

\author{
Shubham Bisen, Parvez Shaik and Vimal Bhatia
}

\begin{abstract}
With the advent of 5G and the need for energyefficient massively connected wireless networks, in this work, we consider an energy harvesting (EH) based multirelay downlink cooperative non-orthogonal multiple access (NOMA) system with practical constraints. The base station serves NOMA users with the help of decode-and-forward based multiple EH relays, where relays harvest the energy from the base station's radio frequency. A relay is selected from the multiple K-relay by using a partial relay selection protocol. The system is considered to operate in halfduplex mode over a generalized independent and identical Nakagami $-m$ fading channel. The closed-form expression of outage probability and ergodic rate are derived for users, under the assumption of imperfect channel state information (CSI) and imperfect successive interference cancellation (SIC) at the receiver node. Expression of outage probability and ergodic rate for both users under the assumption of perfect CSI and perfect SIC are also presented. Further, the asymptotic expression for the outage probability is also shown. The derived analytical expressions are verified through Monte-Carlo simulations.
\end{abstract}

Index Terms-NOMA, Energy harvesting, Nakagami- $m$ fading, outage probability, asymptotic outage, ergodic rate.

\section{INTRODUCTION}

$5^{\text {th }}$ generation $(5 \mathrm{G})$ wireless communication standard is drafted by the third generation partnership project to meet the growing demands of multi-rates data applications. Non-orthogonal multiple access (NOMA) is one of the leading beyond $5 \mathrm{G}$ technologies and is considered a prominent and promising solution with superior spectrum efficiency and massive connectivity [1]-[3]. Recently, cooperative relaying and the NOMA system have gained research attention due to its significant and prominent advantages in improving the coverage and reliability. [4]-[11]. In [5], authors investigated cooperativeNOMA with amplify-and-forward (AF) and decode-andforward (DF) relaying and derived the outage probability and asymptotic outage probability expressions. Considering the fact that multiple relays provide a significant per-

Shubham Bisen, Parvez Shaik and Vimal Bhatia are with the Discipline of Electrical Engineering, Indian Institute of Technology Indore, Indore 453552, India (e-mail: \{phd1901202001; phd1601202003; vbhatia\}@iiti.ac.in.) formance gain in cooperative networks, special attention has been given to the cooperative NOMA with multiple relays and analysis of relay selection techniques [7][10]. In [7], the authors have provided a two-stage maxmin relay selection scheme for the NOMA system with fixed power allocation at relays and different quality of service (QoS) requirements at users. In [7], the users are selected based on different QoS requirements, whereas in [8], authors have considered the users according to their channel condition, with fixed and adaptive power allocation at the relay node. In [9], the outage probability of cooperative NOMA with multiple half-duplex DF relays is analyzed. In [10], the outage probability and sum rate of cooperative NOMA with multiple half-duplex AF relays were studied, considering partial relay selection (PRS). Energy harvesting (EH) from radio frequency signals is considered a viable solution to provide additional lifespan to energy-constrained nodes. Hence, EH based NOMA systems have gained research attention to meet the needs of $5 \mathrm{G}$ and beyond communications. In [12][17], the performance of cooperative NOMA has been studied with EH relaying. A cooperative NOMA with simultaneous wireless information and power transfer is studied in [12], where nearby users acting as EH relays assist the far away NOMA users. In [13], authors analyzed the performance of cooperative NOMA network with EH relaying over Rayleigh fading. In [14], a multiuser NOMA system is analyzed with an EH powered relay node. In [15], the authors evaluated the NOMA system's performance with multiple EH relaying and derived the closed-form expressions of outage probability and ergodic capacity over the Rayleigh fading channel. In [16], the authors analyzed the NOMA system's outage probability with multiple EH AF relays with imperfect CSI and hardware impairment.

In the literature, most of the work assumes perfect knowledge of the channel state information (CSI) at the receiver, which is too idealistic for a practical system. However, in practice, knowledge of perfect CSI is unavailable at the receivers, which leads to channel estimation error (CEE). CEE significantly deteriorates the performance of the system [16], [18], [19]. In [18], 
a closed-form expression of outage probability is derived of a downlink relay aided NOMA system with multiple users over Nakagami- $m$ fading with imperfect CSI.

Contributions: To the best of the author's knowledge, the NOMA system's performance with decode-and-forward (DF) based multiple EH relays over Nakagami- $m$ fading channels has not been considered. Further, a detailed study on the impact of practical constraint, imperfect CSI at receiver nodes, and imperfect SIC is not available in the literature. Nakagami- $m$ fading is considered due to its generalization to a variety of realistic fading channels, which includes Rayleigh fading channel with $m=1$, recently for $\mathrm{THz}$ channels with $m=3$ [20], and also for the unmanned aerial vehicle (UAV) [21]. The main contributions of this work are:

- For the first time in EH based multi-relay NOMA system, we consider the practical case of imperfect CSI at receiver nodes and imperfect SIC, and its impact on the system is analyzed.

- We present a DF based cooperative multiple EH relay based NOMA system employing relay selection and analyze its performance in terms of outage probability by deriving its closed-from expression from the novel end-to-end (e2e) SNR of the considered system for both users for both perfect and imperfect cases.

- Further, we analyzed the system performance at high SNR by deriving the expression of asymptotic outage probability and useful insights are drawn.

- We optimized the EH time fraction parameter $(\alpha)$ to maximize the system throughput. The optimization problem is solved by adopting the particle swarm optimization (PSO) under both perfect and imperfect CSI/SIC conditions.

- Rate analysis of the system is performed by deriving the closed-form expression of ergodic rate for both users and the impact of imperfect CSI and SIC is analyzed.

The rest of the paper is organized as follows: the system model is introduced in Section II. Outage probability analysis is presented in Section III. Asymptotic outage probability analysis is presented in Section IV. Ergodic rate is analyzed in Section V. Numerical and simulation results are discussed in Section VI. Finally, in Section VII, conclusions are drawn.

Notation: $\Gamma(\cdot)$ is gamma function, $G_{p}^{m} \underset{q}{m}\left(\begin{array}{l}a_{1}, \ldots, a_{p} \\ b_{1}, \ldots, b_{p}\end{array} \mid x\right)$ is MeigerG function. Square of the norm is denoted by $|\cdot|^{2}$. Complex Gaussian distribution with mean 0 , variance $\sigma^{2}$ is denoted by $\mathcal{C N}\left(0, \sigma^{2}\right)$ and modified Bessel function of second kind of order $n$ is denoted by $K_{n}(\cdot)$. Expectation operator is denoted by $\mathrm{E}(\cdot)$. Gaussian random variable (RV) with mean $\mu$ and variance $\sigma^{2}$ is represented as $\mathcal{N}\left(\mu, \sigma^{2}\right)$. Probability density function (PDF) and cumulative distribution function (CDF) are

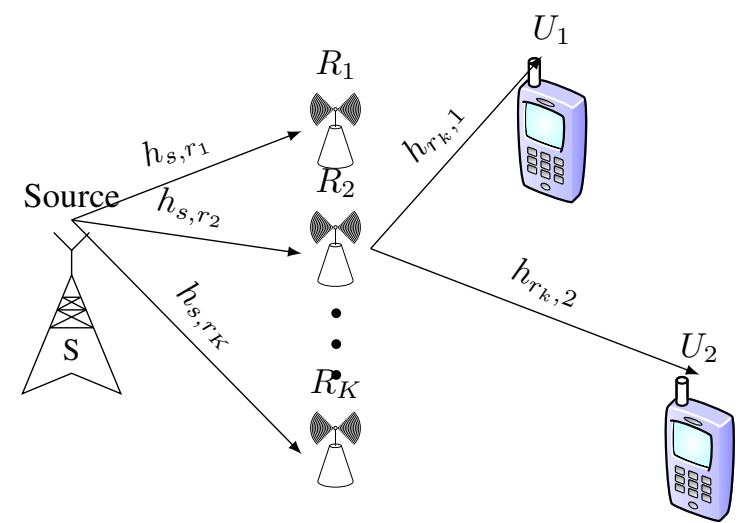

Fig. 1 NOMA system with multiple EH relays

given by $f(\cdot)$ and $F(\cdot)$, respectively. Factorial is denoted by $(\cdot)$ !.

\section{SySTEM MODEL}

We consider a downlink cooperative NOMA system with EH-multiple relays as shown in Fig. 1, where a transmitter $(S)$, i.e., the base station (BS) transmits messages to the downlink users, i.e., $U_{1}$ and $U_{2}$, with the help of cooperative relaying [7], [8]. Both users are ordered according to their channel condition. It is assumed that the weak users $\left(U_{2}\right)$ is with poor channel condition and strong users $\left(U_{1}\right)$ is with good channel condition. To facilitate cooperative relaying, it is also assumed that there are K numbers of relays in the system with DF relaying. Further, it is assumed that each node is equipped with a single antenna and operates in halfduplex mode. All the channel links are considered to be independent and identically distributed (i.i.d), which are modeled by Nakagami $-m$ fading, the channel coefficients corresponding to links are represented as $h_{i, j}$, $i \in\left(s, r_{k}\right)$ and $j \in\left(r_{k}, 1,2\right)$, where the subscript $s, r_{k}, 1$ and 2 represent $\mathrm{BS}, R_{k}, U_{1}$ and $U_{2}$ respectively. The $\left|h_{i, j}\right|$ is assumed to be Nakagami- $m$ with shape parameter $m_{i, j}$ and variance $E\left[\left|h_{i, j}\right|^{2}\right]=\lambda_{i, j}$. Under imperfect CSI, according to minimum mean squared error (MMSE) estimation [22]-[24],

$$
h_{i, j}=\hat{h}_{i, j}+\epsilon_{i, j},
$$

where $h_{i, j}$ is actual channel and $\hat{h}_{i, j}$ is the estimate of the channel $h_{i, j}$, where $h_{i, j}$ and $\hat{h}_{i, j}$ are jointly ergodic and stationary Gaussian process [25]. The $\epsilon_{s, r}$ is the CEE, which is assumed to be complex normal with mean zero and variance $\sigma_{e}^{2}$ [26]. Assuming that $\hat{h}_{i, j}$ and $\epsilon_{i, j}$ are independent 1 , thus the estimated channel variance is given as $\hat{\lambda}_{i, j}=\lambda_{i, j}-\sigma_{e}^{2}$ [27].

In this work, the partial relay selection scheme (PRS) is

\footnotetext{
${ }^{1}$ The independence of $\hat{h}_{i, j}$ and $\epsilon_{i, j}$ was not required, only that they were uncorrelated [22].
} 
considered for the selection of relay node, where the BS selects the relay which provides the best instantaneous channel gain between the BS and relay [28]. The BS continuously monitors the quality of the links between the BS and relays and based on this information, the source selects the best link. The PRS scheme to select the best source to relay link is expressed as $\left|\hat{h}_{s, r}\right|^{2}=$ $\arg \max _{k=1,2, \ldots, K}\left|\hat{h}_{s, r_{k}}\right|^{2}$.

Furthermore, relays harvest the energy from the source transmitted signal. In this paper, we considered the time switching (TS) protocol to harvest energy for $\alpha T$ block time, where $\alpha(0 \leq \alpha \leq 1)$ is the fraction of block time over which relay harvest the energy from a source transmitted signal. The remaining $(1-\alpha) T$ block time is assigned for the information transfer. The information transfer completes in two blocks, the first half of time block $(1-\alpha) \frac{T}{2}$ is assigned for source to relay transmission, and the remaining half $(1-\alpha) \frac{T}{2}$ for relay to users transmission. Thus, the harvested energy for $\alpha T$ time is given by [29] $E_{H}=P_{s} \mu\left|\hat{h}_{s, r}\right|^{2} \alpha T$, where $0 \leq \mu \leq 1$ denotes energy conversion efficiency. The transmit power at $R_{k}$ is expressed as $P_{r}=\frac{E_{H}}{(1-\alpha) T / 2}=$ $\frac{2 P_{s} \mu\left|\hat{h}_{s, r}\right|^{2} \alpha}{1-\alpha}$. Initially, BS transmits the NOMA signal for users by performing power domain multiplexing and superposition coding. The transmitted signal is given by $x_{s}=\sum_{i=1}^{2} \sqrt{a_{i}} x_{i}$, where, $x_{i}$ denotes complex modulated symbols with unit energy for $U_{i}$ (i.e., $\mathrm{E}\left\{\left|x_{i}\right|^{2}\right\}=$ $1)$. Further, $a_{i}$ is the power allocation coefficients for $x_{i}$ with $\sum_{i=1}^{2} a_{i}=1$ and $a_{i}>0$. The received signal at $R_{k}$ is given by $y_{r_{k}}=\left(\hat{h}_{s, r}+\epsilon_{s, r_{k}}\right) \sqrt{P_{s}} x_{s}+z_{s, r_{k}}$, where, $P_{s}$ indicates the total transmit power at BS, $z_{s, r}$ represents additive white Gaussian noise (AWGN) with zero mean and variance $\sigma_{0}^{2}$. As DF transmission protocol is employed at the relay, $R_{k}$ has to first decode both $x_{1}$ and $x_{2}$ before transmitting. The signal-to-interference noise ratio (SINR) at $R_{k}$ to decode $x_{2}$ in the presence of imperfect CSI is given by

$$
\gamma_{r_{k}, 2}=\frac{\left|\hat{h}_{s, r}\right|^{2} P_{s} a_{2}}{\left|\hat{h}_{s, r}\right|^{2} P_{s} a_{1}+P_{s} \sigma_{e}^{2}+\sigma_{0}^{2}} .
$$

According to SIC principle, $x_{1}$ is decoded by removing $x_{2}$ from $y_{r_{k}}$, the SIC is perfect, $x_{2}$ will be completely removed. Otherwise decoding of $x_{1}$ will be carried out in the presence of residual interference due to imperfect SIC. Thus SINR in the presence of imperfect CSI and imperfect SIC at $R_{k}$ to decode $x_{1}$ is given by

$$
\gamma_{r_{k}, 1}=\frac{\left|\hat{h}_{s, r}\right|^{2} P_{s} a_{1}}{\left|\hat{h}_{s, r}\right|^{2} \beta P_{s} a_{2}+P_{s} a_{1} \sigma_{e}^{2}+P_{s} \beta a_{2} \sigma_{e}^{2}+\sigma_{0}^{2}} .
$$

where, $\beta$ represents the residual interference due to imperfect SIC, $0 \leq \beta \leq 1$, and $\beta=0$ refer to perfect SIC. After decoding $x_{2}$ and $x_{1}, R_{k}$ will re-encode the information bit using superposition coding. Thus the transmitted signal by $R_{k}$ is given by $x_{r}=\sum_{i=1}^{2} \sqrt{a_{i}} x_{i}$. The received signal at $U_{1}$ and $U_{2}$ is given as $y_{l}=$ $\left(\hat{h}_{r_{k}, l}+\epsilon_{r_{k}, l}\right) \sqrt{P_{r}} x_{r}+z_{r_{k}, l}, l=1,2$. Where $P_{r}$ is harvested power at $R_{k}, z_{r_{k}, l}$ represent AWGN with zero mean and variance $\sigma_{0}^{2}$. Thereafter, $U_{1}$ will performs SIC to obtain its own symbol $x_{1}$. Thus, the SINR at $U_{1}$ to decode $x_{2}$ and $x_{1}$ under imperfect CSI and imperfect $\mathrm{SCI}$ are given as

$$
\begin{gathered}
\gamma_{1,2}=\frac{\left|\hat{h}_{r_{k}, 1}\right|^{2} P_{r} a_{2}}{\left|\hat{h}_{r_{k}, 1}\right|^{2} P_{r} a_{1}+P_{r} \sigma_{e}^{2}+\sigma_{0}^{2}}, \\
\gamma_{1,1}=\frac{\left|\hat{h}_{r_{k}, 1}\right|^{2} P_{r} a_{1}}{\left|\hat{h}_{r_{k}, 1}\right|^{2} \beta P_{r} a_{2}+P_{r} a_{1} \sigma_{e}^{2}+P_{r} \beta a_{1} \sigma_{e}^{2}+\sigma_{0}^{2}} .
\end{gathered}
$$

The SINR to decode $x_{2}$ at $U_{2}$ under imperfect CSI is given by

$$
\gamma_{2,2}=\frac{\left|\hat{h}_{r_{k}, 2}\right|^{2} P_{r} a_{2}}{\left|\hat{h}_{r_{k}, 2}\right|^{2} P_{r} a_{1}+P_{r} \sigma_{e}^{2}+\sigma_{0}^{2}} .
$$

\section{Outage Probability Analysis}

In this section, we derive the outage probability expression for both NOMA users. Outage probability gives the achievable maximum rate for error-free transmission [30]. The closed-form outage probability expressions for $U_{1}$ and $U_{2}$ under imperfect CSI/ SCI and perfect CSI/SIC are obtained in the following subsections.

\section{A. Outage probability of $U_{1}$ under imperfect CSI and imperfect SIC}

$U_{1}$ is said to be in outage, when $U_{1}$ fails to detect either of the two symbols $x_{1}$ and $x_{2}$ at $R_{k}$ and $U_{1}$. Therefore the outage probability of $U_{1}$ is defined as

$$
\begin{aligned}
P_{\text {out }, 1}= & \operatorname{Pr}\left\{\gamma_{r_{k}, 2}<\gamma_{t h 2}, \gamma_{r_{k}, 1}<\gamma_{t h 1},\right. \\
& \left.\gamma_{1,2}<\gamma_{t h 2}, \gamma_{1,1}<\gamma_{t h 1}\right\},
\end{aligned}
$$

where, $\gamma_{t h 1}$ and $\gamma_{t h 2}$ are predefined SINR threshold. $\gamma_{t h 1}$ and $\gamma_{t h 2}$ can be represented as $\gamma_{t h 1}=2^{\frac{2 r_{1}}{1-\alpha}}-1$ and $\gamma_{t h 2}=2^{\frac{2 r_{2}}{1-\alpha}}-1$, where, $r_{1}$ and $r_{2}$ are the desired target rates.

Lemma 1. When $\gamma_{t h 2}<\frac{a_{2}}{a_{1}}, \gamma_{t h 1}<\frac{a_{1}}{\beta a_{2}}, \lambda_{2}>\lambda_{1}$ and $\Omega_{1}>\Omega_{2}$. The outage probability of $U_{1}$ under imperfect CSI and imperfect SIC is defined in (8)

In (8), $A_{1}=\frac{K}{\left(m_{s, r_{k}}-1\right) !} \sum_{n=0}^{m_{r_{k}, 1}-1} \sum_{j=0}^{n} \frac{1}{n !} \hat{\beta}_{r_{k}, 1}^{n}\left(\begin{array}{c}n \\ j\end{array}\right)$, $A_{2}=\sum_{k=1}^{K-1} \bigcup_{k} \Xi_{1, k} \Xi_{2, k}(-1)^{k}\left(\begin{array}{c}K-1 \\ k\end{array}\right), \kappa_{1}=m_{s, r_{k}}-$ $j-l_{1}, \kappa_{2}=m_{s, r_{k}}-j-l_{2}+\bar{i}, \lambda_{1}=\frac{\gamma_{t h 2} \sigma_{0}^{2}(1-\alpha)}{\left(a_{2}-a_{1} \gamma_{t h 2}\right) \alpha \mu 2 \rho}$, $\lambda_{2}=\frac{\gamma_{t h 1}(1-\alpha)}{\left(a_{1}-a_{2} \beta \gamma_{t h 1}\right) \alpha \mu 2 \rho}, \Omega_{1}=\frac{\gamma_{t h 2} \sigma_{e}^{2}}{a_{2}-a_{1} \gamma_{t h 2}}, \Omega_{2}=$ $\frac{\gamma_{t h 2}\left(a_{1}+a_{2} \beta\right) \sigma_{e}^{2}}{a_{1}-a_{2} \beta \gamma_{t h 2}}, \gamma=\max \left[\psi,\left(\frac{\lambda_{2}-\lambda_{1}}{\Omega_{1}-\Omega_{2}}\right)\right], \rho=$ $\frac{P_{s}}{\sigma_{0}^{2}}, \hat{\beta}_{r_{k}, 1}=\frac{m_{r_{k}, 1}}{\hat{\lambda}_{r_{k}, 1}}, \hat{\beta}_{s, r_{k}}=\frac{m_{s, r_{k}}}{\hat{\lambda}_{s, r_{k}}}, \psi=$ $\max \left[\Delta_{1}, \frac{\gamma_{t h 1}\left(\sigma_{e}^{2}\left(a_{1}+a_{2} \beta\right)+(1 / \rho)\right)}{\left(a_{1}-a_{2} \beta \gamma_{t h 1}\right)}\right], \Delta_{1}=$ $\frac{\gamma_{t h 2}\left(\sigma_{e}^{2}+(1 / \rho)\right)}{\left(a_{2}-a_{1} \gamma_{t h 2}\right)}, \iota_{1}=\sum_{l_{1}=0}^{N_{t}} \frac{(-1)^{l_{1}}}{l_{1} !}, \iota_{2}=\sum_{l_{2}=0}^{N_{t}} \frac{(-1)^{l_{2}}}{l_{2} !}$, 


$$
\begin{aligned}
P_{\text {out }, 1}= & 1-\left[e ^ { - \Omega _ { 1 } \hat { \beta } _ { r _ { k } , 1 } } A _ { 1 } \Omega _ { 1 } ^ { n - j } \lambda _ { 1 } ^ { j } \left[\iota_{1}\left(\hat{\beta}_{r_{k}, 1} \lambda_{1}\right)^{l_{1}} \hat{\beta}_{s, r_{k}}^{\left(j+l_{1}\right)} \Gamma\left(\kappa_{1}, \hat{\beta}_{s, r_{k}} \gamma\right)+A_{2}\left(\hat{\beta}_{r_{k}, 1} \lambda_{1}\right)^{l_{2}} \hat{\beta}_{s, r_{k}}^{\left(j+l_{2}-\bar{i}\right)} \Gamma\left(\kappa_{2}, \hat{\beta}_{s, r_{k}}(k+1) \gamma\right)\right.\right. \\
& \left.\iota_{2}(k+1)^{-\kappa_{2}}\right]+e^{-\Omega_{2} \hat{\beta}_{r_{k}, 1}} A_{1} \Omega_{2}^{n-j} \lambda_{1}^{j}\left[\iota_{1}\left(\hat{\beta}_{r_{k}, 1} \lambda_{1}\right)^{l_{1}} \hat{\beta}_{s, r_{k}}^{\left(j+l_{1}\right)}\left[\Gamma\left(\kappa_{1}, \hat{\beta}_{s, r_{k}} \psi\right)-\Gamma\left(\kappa_{1}, \frac{\hat{\beta}_{s, r_{k}}\left(\lambda_{2}-\lambda_{1}\right)}{\Omega_{1}-\Omega_{2}}\right)\right]\right. \\
& \left.\left.+A_{2} \iota_{2}\left(\hat{\beta}_{r_{k}, 1} \lambda_{1}\right)^{l_{2}}(k+1)^{-\kappa_{2}} \hat{\beta}_{s, r_{k}}^{\left(j+l_{2}-\bar{i}\right)}\left[\Gamma\left(\kappa_{2},(k+1) \hat{\beta}_{s, r_{k}} \psi\right)-\Gamma\left(\kappa_{2}, \frac{(k+1) \hat{\beta}_{s, r_{k}}\left(\lambda_{2}-\lambda_{1}\right)}{\left(\Omega_{1}-\Omega_{2}\right)}\right)\right]\right]\right]
\end{aligned}
$$

$\bigcup_{k}=\sum_{i_{1}}^{k} \sum_{i_{2}}^{k-i_{2}} \ldots \sum_{i_{m_{s}, r_{k}}}^{k-i_{1}^{\prime \prime \prime} i_{m_{s}, r_{k}}}-2$

$\Xi_{1, k} \quad=\quad\left(\begin{array}{c}k \\ i_{1}\end{array}\right) \ldots\left(\begin{array}{c}k-i_{1}-\ldots i_{m_{s}, r_{k}} \\ i_{m_{s, r_{k}}}\end{array}\right), \Xi_{2, k}=$

$\prod_{n=0}^{m_{s, r_{k}}-2}\left(\frac{\hat{\beta}_{s, r_{k}}^{n}}{n !}\right)^{i_{n}+1}\left(\frac{\hat{\beta}_{s, r_{k}}^{m_{s}, r_{k}-1}}{\left(m_{s, r_{k}}-1\right) !}\right)^{k-i_{1}-\ldots i_{m_{s}, r_{k}}-1}$

and $\bar{i}=\left(m_{s, r_{k}}-1\right)\left(k-i_{1}\right)-\left(m_{s, r_{k}}-2\right) i_{2}-\left(m_{s, r_{k}}-\right.$ 3) $i_{3}-\ldots i_{m_{s, r_{k}}-1}$. Further, when $\gamma_{t h 2}>\frac{a_{2}}{a_{1}}, \gamma_{t h 1}>\frac{a_{1}}{\beta a_{2}}$, $P_{\text {out }, 1}=1$. Proof: See Appendix A

B. Outage probability of $U_{2}$ under imperfect CSI

$U_{2}$ is said to be in outage, when $U_{2}$ fails to detect $x_{2}$ at $R_{k}$ and $U_{2}$.

$$
P_{\text {out }, 2}=\operatorname{Pr}\left\{\gamma_{r_{k}, 2}<\gamma_{t h 2}, \gamma_{2,2}<\gamma_{t h 2}\right\},
$$

Lemma 2. The outage probability of $U_{2}$ under imperfect CSI is given as

$$
\begin{aligned}
P_{\text {out }, 2}= & 1-e^{-\Omega_{1} \hat{\beta}_{r_{k}, 2}} A_{3} \Omega_{1}^{n-j} \lambda_{1}^{j}\left[\iota_{1}\left(\hat{\beta}_{r_{k}, 2} \lambda_{1}\right)^{l_{1}} \hat{\beta}_{s, r_{k}}^{\left(j+l_{1}\right)}\right. \\
& \Gamma\left(\kappa_{1}, \hat{\beta}_{s, r_{k}} \Delta_{1}\right)+A_{2} \iota_{2}\left(\hat{\beta}_{r_{k}, 2} \lambda_{1}\right)^{l_{2}} \hat{\beta}_{s, r_{k}}^{\left(j+l_{2}-\bar{i}\right)} \\
& \left.(k+1)^{-\kappa_{2}} \Gamma\left(\kappa_{2},(k+1) \hat{\beta}_{s, r_{k}} \Delta_{1}\right)\right],
\end{aligned}
$$

where $A_{3}=\frac{K}{\left(m_{s, r_{k}}-1\right) !} \sum_{n=0}^{m_{r_{k}, 2}-1} \sum_{j=0}^{n} \frac{1}{n !}\left(\frac{m_{r_{k}, 2}}{\lambda_{r_{k}, 2}}\right)^{n}$ $\left(\begin{array}{c}n \\ j\end{array}\right)$. Further when $\gamma_{t h 2}>\frac{a_{2}}{a_{1}}, P_{\text {out }, 2}=1$.

Proof: See Appendix B

C. Outage probability of $U_{1}$ under perfect CSI and perfect $S I C$

The outage probability of $U_{1}$ under perfect CSI and perfect SIC is obtained by considering the perfect channel estimation and perfect SIC at all nodes. The $\left|\hat{h}_{i, j}\right|^{2}=$ $\left|h_{i, j}\right|^{2}$ i.e., the CEE $\epsilon_{i, j}=0$ and residual interference due to imperfect SIC $\beta=0$.

Lemma 3. The outage probability of $U_{1}$ under perfect CSI/SIC is expressed as

$$
\begin{aligned}
P_{\text {out }, 1}^{P}= & 1-B_{1}\left(\beta_{r_{k}, 1} \Delta_{2}\right)^{n}\left[\iota_{1}\left(\beta_{r_{k}, 1} \Delta_{2}\right)^{l_{1}}\right. \\
& \Gamma\left(\kappa_{3}, \beta_{s, r_{k}} \Delta_{3}\right) \beta_{s, r_{k}}^{\left(n+l_{1}\right)}+A_{2} \iota_{2}\left(\beta_{r_{k}, 1} \Delta_{2}\right)^{l_{2}} \\
& \left.(k+1)^{-\kappa_{4}} \beta_{s, r_{k}}^{\left(n+l_{2}-\bar{i}\right)} \Gamma\left(\kappa_{4},(k+1) \beta_{s, r_{k}} \Delta_{3}\right)\right],
\end{aligned}
$$

where $B_{1}=\frac{K}{\left(m_{s, r_{k}}-1\right) !} \sum_{n=0}^{m_{r_{k}, 1}-1} \frac{1}{n !}, \kappa_{3}=m_{s, r_{k}}-$ $n-l_{1}, \quad \kappa_{4}=m_{s, r_{k}}-n-l_{2}+\bar{i}, \Delta_{3}=$ $\max \left[\frac{\gamma_{t h 2}}{\left(a_{2}-a_{1} \gamma_{t h 2}\right) \rho}, \frac{\gamma_{t h 1}}{a_{1} \rho}\right], \Delta_{2}=\max \left[\lambda_{1}, \frac{\gamma_{t h 1}(1-\alpha)}{a_{1} \alpha 2 \mu \rho}\right]$.

Proof: See Appendix C

\section{Outage probability of $U_{2}$ under perfect CSI}

The outage probability of $U_{2}$ under perfect CSI is obtained by considering the perfect CSI assumption as used in $P_{\text {out }, 1}^{P}$. Thus the outage probability of $U_{2}$ under perfect CSI is given as

$$
\begin{aligned}
P_{\text {out }, 2}^{P}= & 1-B_{2}\left(\beta_{r_{k}, 2} \lambda_{1}\right)^{n}\left[\iota_{1}\left(\beta_{r_{k}, 2} \lambda_{1}\right)^{l_{1}}\left(\beta_{s, r_{k}}\right)^{n+l_{1}}\right. \\
& \Gamma\left(\kappa_{3}, \chi\right)+A_{2} \iota_{2}(k+1)^{\kappa_{4}}\left(\beta_{r_{k}, 2} \lambda_{1}\right)^{l_{2}} \\
& \left.\left(\beta_{s, r_{k}}\right)^{n+l_{2}-\bar{i}} \Gamma\left(\kappa_{4},(k+1) \chi\right)\right]
\end{aligned}
$$

where $\quad B_{2}=\frac{K}{\left(m_{s, r_{k}}-1\right) !} \sum_{n=0}^{m_{r_{k}, 2}-1} \frac{1}{n !}, \quad \chi=$ $\frac{m_{s, r_{k}} \gamma_{t h 2}}{\lambda_{s, r_{k}}\left(a_{2}-a_{1} \gamma_{t h 2}\right) \rho}$. Further, when $\gamma_{t h 2}>\frac{a_{2}}{a_{1}}, P_{o u t, 2}^{P}=1$.

\section{Asymptotic Outage Probability}

In this section, an approximation of the outage probability at a high SNR region is provided. The asymptotic outage probability is obtained by considering the $\rho \rightarrow \infty$. Further, at high SNR, the approximated CDF is given as $F(x) \underset{x \rightarrow 0}{\approx} \frac{1}{m_{i, j} !}\left(\beta_{i, j} x\right)^{m_{i, j}}$ [16].

A. Asymptotic outage probability of $U_{1}$ under imperfect CSI and imperfect SIC

At high SNR, the asymptotic outage probability of $U_{1}$ is obtained by utilizing the approximated CDF equation in (A.2) and equating $\frac{1}{\rho} \rightarrow 0$ in $\lambda_{1}$ and $\lambda_{2}$. The asymptotic outage probability of $U_{1}$ under imperfect CSI/SIC is given as

$$
\begin{aligned}
P_{\text {out }, 1}^{a s y}= & -\left[\left(1-\frac{1}{m_{s, r_{k}} !}\left(\hat{\beta}_{s, r_{k}} \psi\right)^{m_{s, r_{k}}}\right)^{K}\right. \\
& \left.e^{-\hat{\beta}_{r_{k}, 1} a} \sum_{n=0}^{m_{r_{K}, 1}-1} \frac{1}{n !}\left(\hat{\beta}_{r_{k}, 1} a\right)^{n}\right]
\end{aligned}
$$

where, $a=\max \left[\Omega_{1}, \Omega_{2}\right]$. 
B. Asymptotic outage probability of $U_{2}$ under imperfect CSI

The asymptotic outage probability of $U_{2}$ is obtained by approximating CDF equation and equating $\frac{1}{\rho} \rightarrow 0$ in $\lambda_{1}$. The asymptotic outage probability of $U_{2}$ under imperfect CSI is given as

$$
\begin{aligned}
P_{\text {out }, 2}^{a s y}= & -\left[\left(1-\frac{1}{m_{s, r_{k}}}\left(\hat{\beta}_{s, r_{k}} \Delta_{1}\right)^{m_{s, r_{k}}}\right)^{K}\right. \\
& \left.e^{-\hat{\beta}_{r_{k}, 2} \Omega_{1}} \sum_{n=0}^{m_{r_{K}, 2}-1} \frac{1}{n !}\left(\hat{\beta}_{r_{k}, 2} \Omega_{1}\right)^{n}\right] .
\end{aligned}
$$

C. Asymptotic outage probability of $U_{1}$ under perfect CSI and perfect SIC

The asymptotic outage probability of $U_{1}$ under perfect CSI and SIC is obtained by utilizing the approximated CDF. The asymptotic outage probability of $U_{1}$ under perfect CSI/SIC is given by

$$
\begin{aligned}
& P_{\text {out }, 1}^{P, a s y}=\left(\frac{1}{m_{s, r_{k}} !}\left(\beta_{s, r_{k}} \Delta_{3}\right)^{m_{s, r_{k}}}\right)^{K}+\frac{\left(\beta_{r_{k}, 1} \Delta_{2}\right)^{m_{r_{k}, 1}}}{\left(m_{s, r_{k}}-1\right) !} \\
& \frac{K}{m_{r_{k}, 1} !}\left[\beta_{s, r_{k}}^{m_{r_{k}, 1}} \Gamma\left(\kappa_{5}, \beta_{s, r_{k}} \Delta_{3}\right)+A_{2} \beta_{s, r_{k}}^{\left(m_{r_{k}, 1}-\bar{i}\right)}\right. \\
&\left.(k+1)^{-\left(\kappa_{5}+\bar{i}\right)} \Gamma\left(\kappa_{5}+\bar{i},(k+1) \beta_{s, r_{k}} \Delta_{1}\right)\right] .
\end{aligned}
$$

where $\kappa_{5}=m_{s, r_{k}}-m_{r_{k}, 1}$.

D. Asymptotic outage probability of $U_{2}$ under perfect CSI

The asymptotic outage probability of $U_{2}$ under perfect CSI is obtained by approximating CDF. The asymptotic outage probability of $U_{2}$ under perfect CSI is given by

$$
\begin{aligned}
P_{\text {out }, 2}^{P, a s y}= & \left(\frac{1}{m_{s, r_{k}} !} \chi^{m_{s, r_{k}}}\right)^{K}+\frac{K\left(\beta_{r_{k}, 2} \lambda_{1}\right)^{m_{r_{k}, 2}}}{\left(m_{s, r_{k}}-1\right) ! m_{r_{k}, 2} !} \\
& {\left[\beta_{s, r_{k}, 2}^{m_{r_{k}}} \Gamma\left(\kappa_{6}, \chi\right)+A_{2}(k+1)^{-\left(\kappa_{6}+i\right)}\right.} \\
& \left.\left(\beta_{s, r_{k}}\right)^{m_{r_{k}, 2}-\bar{i}} \Gamma\left(\kappa_{6}+\bar{i},(k+1) \chi\right)\right] .
\end{aligned}
$$

where $\kappa_{6}=m_{s, r_{k}}-m_{r_{k}, 2}$.

\section{E. Optimization of fraction of EH time $(\alpha)$}

In this section, fraction of $\mathrm{EH}$ time $(\alpha)$ is optimized in order to maximize the throughput of the system. The system throughput of a dual-hop system in a delaylimited transmission mode for a fixed transmission rate from the outage probability is given as [31]

$$
\tau=\frac{\left(1-P_{\text {out }, 1}\right) r_{1}(1-\alpha)}{2}+\frac{\left(1-P_{\text {out }, 2}\right) r_{2}(1-\alpha)}{2},
$$

where $\lambda_{t h 1}=2^{\frac{2 r_{1}}{1-\alpha}}-1$ and $\lambda_{t h 2}=2^{\frac{2 r_{2}}{1-\alpha}}-1$ are the threshold SNR for a fixed rate $r_{1}$ and $r_{2}$ respectively. Maximum throughput can be attained by optimizing $\alpha$.
The objective function for maximizing throughput can be formulated as follows

$$
\begin{aligned}
& \alpha^{*}=\arg \max _{\alpha} \tau \text { subject to } 0<\alpha<1, \\
& \alpha^{*}=\arg \max _{\alpha} \frac{\left(1-P_{\text {out }, 1}\right) R_{1}(1-\alpha)}{2} \\
&+\frac{\left(1-P_{\text {out }, 2}\right) R_{2}(1-\alpha)}{2}
\end{aligned}
$$$$
\text { subject to } 0<\alpha<1
$$

The objective function in (18) is nonlinear and nonconvex. Thus, we propose a low-complexity optimum fraction of EH time that maximize the system throughput based on a PSO algorithm [32], wherein the optimal solution from each iteration in the search space is based on swarm of particles. It is noteworthy that we choose PSO because it offers fast convergence and stability [33], [34]. The algorithm for determining the PSO-based solution is shown in Algorithm 1. Let $\tau(\alpha)$ denote the objective value of solution $\alpha$ as given in (17). Let $\alpha_{i}$ denotes the position of particle $i\left(1 \leq i \leq M A X_{\text {particles }}\right)$, where $M A X_{\text {particles }}$ denotes the number of particles.

\section{ERgodic RATE}

In this section, we derive the ergodic rate expression for NOMA users. The achievable rate for error-free transmission is given as

$$
\begin{aligned}
R_{i} & =E\left[\frac{1-\alpha}{2} \log _{2}\left(1+\gamma_{i, j}\right)\right], \\
& =\frac{1-\alpha}{2 \ln 2} \int_{z=0}^{\infty} \frac{1-F_{i, j}(z)}{1+z} d z .
\end{aligned}
$$

where $F_{i, j}$ represents CDF of SNR $\gamma_{i, j}$. The closed-form ergodic rate expressions for $U_{1}$ and $U_{2}$ are obtained in the following subsections.

\section{A. Ergodic rate of $U_{1}$ under imperfect CSI and imperfect SIC}

The ergodic rate of $U_{1}$ is defined as

$$
\begin{aligned}
R_{1}= & E\left[\frac{1-\alpha}{2} \log _{2}\left(1+\min \left(\gamma_{r_{k}, 1}, \gamma_{1,1}\right)\right)\right], \\
R_{1}= & E\left[\frac{1-\alpha}{2} \log _{2}(1+\min (\right. \\
& \frac{\left|\hat{h}_{s, r}\right|^{2} P_{s} a_{1}}{\left|\hat{h}_{s, r}\right|^{2} \beta P_{s} a_{2}+P_{s} \sigma_{e}^{2}\left(\beta a_{2}+a_{1}\right)+\sigma_{0}^{2}}, \\
& \left.\left.\frac{\left|\hat{h}_{r_{k}, 1}\right|^{2} P_{r} a_{1}}{\left|\hat{h}_{r_{k}, 1}\right|^{2} \beta P_{r} a_{2}+P_{r} \sigma_{e}^{2}\left(\beta a_{2}+a_{1}\right)+\sigma_{0}^{2}}\right)\right] .
\end{aligned}
$$

Considering the practical scenario, the harvested energy at the relay is always small. Hence the transmit power of the relay is much lower than that of the source. Thus 


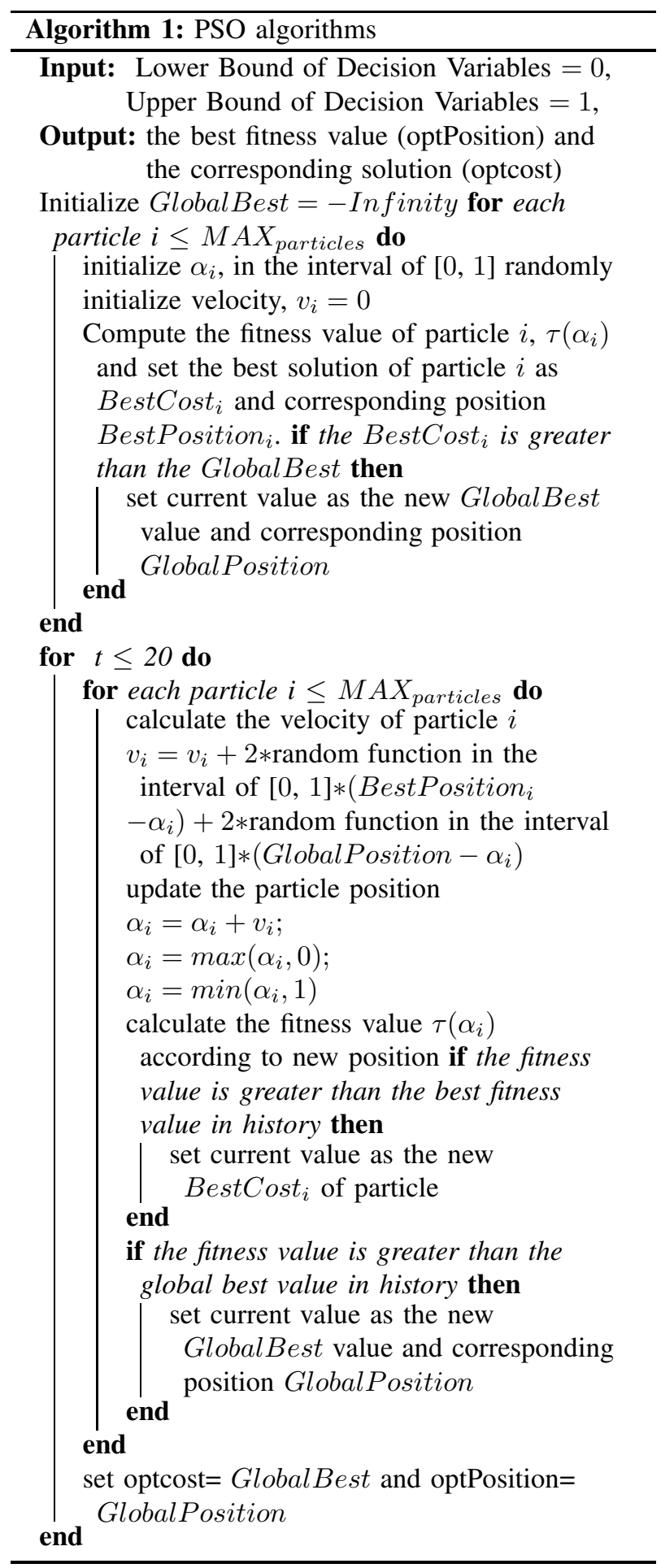

it is assumed that the SINR at the destinations is lower than the SINR at the relay [15].

$$
\begin{gathered}
\frac{\left|\hat{h}_{s, r}\right|^{2} P_{s} a_{1}}{\left|\hat{h}_{s, r}\right|^{2} \beta P_{s} a_{2}+P_{s} \sigma_{e}^{2}\left(\beta a_{2}+a_{1}\right)+\sigma_{0}^{2}}> \\
\frac{\left|\hat{h}_{r_{k}, 1}\right|^{2} P_{r} a_{1}}{\left|\hat{h}_{r_{k}, 1}\right|^{2} \beta P_{r} a_{2}+P_{r} \sigma_{e}^{2}\left(\beta a_{2}+a_{1}\right)+\sigma_{0}^{2}} .
\end{gathered}
$$

Therefore, (21) will reduce to

$$
\begin{aligned}
R_{1}= & E\left[\frac{1-\alpha}{2} \log _{2}(1+\right. \\
& \left.\left.\frac{\left|\hat{h}_{r_{k}, 1}\right|^{2} P_{r} a_{1}}{\left|\hat{h}_{r_{k}, 1}\right|^{2} \beta P_{r} a_{2}+P_{r} \sigma_{e}^{2}\left(\beta a_{2}+a_{1}\right)+\sigma_{0}^{2}}\right)\right],
\end{aligned}
$$

After some manipulation and by using (19), the above equation can be further simplified as

$$
\begin{aligned}
R_{1}= & \frac{1-\alpha}{2 \ln 2} \int_{z=0}^{\infty} \frac{1-F_{P}(z)}{1+z} d z \\
& -\frac{1-\alpha}{2 \ln 2} \int_{z=0}^{\infty} \frac{1-F_{Q}(z)}{1+z} d z,
\end{aligned}
$$

where $P=\left|\hat{h}_{r_{k}, 1}\right|^{2} P_{r}\left(\beta a_{2}+a_{1}\right)+P_{r} \sigma_{e}^{2}\left(\beta a_{2}+a_{1}\right)$ and $Q=\left|\hat{h}_{r_{k}, 1}\right|^{2} \beta P_{r} a_{2}+P_{r} \sigma_{e}^{2}\left(\beta a_{2}+a_{1}\right)$. After some manipulation and using [35, eq.(3.471.9)], the CDF of $P$ and $\mathrm{Q}$ are given as follow

$$
\begin{aligned}
F_{P}(z)=1 & -2 e^{\hat{\beta}_{r_{k}, 1} \sigma_{e}^{2}} A_{1}\left(-\sigma_{e}^{2}\right)^{n-j} \hat{\beta}_{s, r_{k}}^{m_{s, r_{k}}}\left(\frac{z}{\zeta}\right)^{j} \\
& {\left[\left(\frac{\hat{\beta}_{r_{k}, 1} z}{\hat{\beta}_{s, r_{k} \zeta} \zeta}\right)^{\frac{\tau_{1}}{2}} K_{\tau_{1}}\left(2 \sqrt{\xi_{1} z}\right)+A_{2}\left(\frac{\hat{\beta}_{r_{k}, 1}}{\hat{\beta}_{s, r_{k}}}\right)^{\frac{\tau_{1}+\bar{i}}{2}}\right.} \\
& \left.\left(\frac{z}{\zeta(k+1)}\right)^{\frac{\tau_{1}+\bar{i}}{2}} K_{\tau_{1}+\bar{i}}\left(2 \sqrt{\xi_{1} z(k+1)}\right)\right],
\end{aligned}
$$

where $\tau_{1}=m_{s, r_{k}}-j, \xi_{1}=\frac{\hat{\beta}_{r_{k}, 1} \hat{\beta}_{s, r_{k}}}{\zeta}$ and $\zeta=$ $\frac{2 \rho \mu \alpha\left(a_{1}+\beta a_{2}\right)}{1-\alpha}$.

$$
\begin{aligned}
F_{Q}(z)= & 1-2 e^{\hat{\beta}_{r_{k}, 1} \xi_{3}} A_{1}\left(-\xi_{3}\right)^{n-j} \hat{\beta}_{s, r_{k}}^{m_{s, r_{k}}}\left(\frac{z}{\bar{\zeta}}\right)^{j} \\
& {\left[K_{\tau_{1}}\left(2 \sqrt{\xi_{2} z}\right)\left(\frac{\hat{\beta}_{r_{k}, 1} z}{\hat{\beta}_{s, r_{k}} \bar{\zeta}}\right)^{\frac{\tau_{1}}{2}}+A_{2}\left(\frac{\hat{\beta}_{r_{k}, 1}}{\hat{\beta}_{s, r_{k}}}\right)^{\frac{\tau_{1}+\bar{i}}{2}}\right.} \\
& \left.\left(\frac{z}{\bar{\zeta}(k+1)}\right)^{\frac{\tau_{1}+\bar{i}}{2}} K_{\tau_{1}+\bar{i}}\left(2 \sqrt{\xi_{2} z(k+1)}\right)\right],
\end{aligned}
$$

where $\xi_{2}=\frac{\hat{\beta}_{r_{k}, 1} \hat{\beta}_{s, r_{k}}}{\bar{\zeta}}, \xi_{3}=\frac{\sigma_{e}^{2}\left(a_{1}+\beta a_{2}\right)}{\beta a_{2}}$ and $\bar{\zeta}=$ $\frac{2 \rho \mu \alpha \beta a_{2}}{1-\alpha}$. Further, substituting (25) and (26) in (24), and using [35, eq. (7.811.5), (9.34.3)], after some manipulation the ergodic rate of $U_{1}$ is given as

$$
R_{1}=\frac{1-\alpha}{2 \ln 2} I_{1}
$$

where $I_{1}$ is defined in (32). 


\section{B. Ergodic rate of $U_{2}$ under imperfect $C S I$}

The ergodic rate of $U_{2}$ is define as

$$
R_{2}=E\left[\frac{1-\alpha}{2} \log _{2}\left(1+\min \left(\gamma_{r_{k}, 2}, \gamma_{2,2}\right)\right)\right],
$$

Substituting (2) and (6) in (28), and by employing the same approach as of $U_{1}$, the ergodic rate of $U_{2}$ is given by

$$
R_{2}=\frac{1-\alpha}{2 \ln 2} J_{1},
$$

where $J_{1}$ is defined in (33). where $\nu=\frac{2 \rho \mu \alpha}{1-\alpha}$ and $\bar{\nu}=$ $\frac{2 \rho a_{1} \mu \alpha}{1-\alpha}$.

C. Ergodic rate of $U_{1}$ under perfect CSI and perfect SIC

The ergodic rate of $U_{1}$ is obtained by substituting the SINR under the assumption of perfect CSI/SIC in (20). The ergodic rate of $U_{1}$ under perfect CSI/SIC is given as

$$
\begin{aligned}
R_{1}^{P}= & \frac{1-\alpha}{2 \ln 2} B_{1}\left[G_{3}^{1} \underset{1}{3} \underset{0, m_{s, r_{k}, n}^{0}}{0} \mid \xi_{4}\right)+A_{2} \beta_{s, r_{k}}^{-\bar{i}} \\
& \left.\left(\frac{1}{k+1}\right)^{m_{s, r_{k}}+\bar{i}} G_{3}^{1} \underset{1}{1}\left({ }_{0, m_{s, r_{k}}+\bar{i}, n}^{0} \mid \xi_{4}\right)\right] .
\end{aligned}
$$

where $\xi_{4}=\frac{\beta_{s, r_{k}} \beta_{r_{k}, 1}}{\bar{\nu}}$.

\section{Ergodic rate of $U_{2}$ under perfect CSI}

The ergodic rate of $U_{2}$ is obtained by substituting the SINR under the perfect CSI assumption in (28). The ergodic rate of $U_{2}$ under perfect CSI is given as

$$
\begin{aligned}
& R_{2}^{P}=\frac{1-\alpha}{2 \ln 2}\left[B_{2}\left(G_{3}^{1}{ }_{1}^{3} \underset{0, m_{s, r_{k}}, n}{0} \mid \xi_{5}\right)+A_{2} \beta_{s, r_{k}}^{-\bar{i}}\right. \\
& \left.\left(\frac{1}{k+1}\right)^{m_{s, r_{k}}+\bar{i}} G_{3}^{1}{ }_{1}^{3}\left(\underset{0, m_{s, r_{k}}+\bar{i}, n}{0} \mid \xi_{5}(k+1)\right)\right) \\
& -B_{2}\left(G_{3}^{1}{ }_{1}^{3}\left(\underset{0, m_{s, r_{k}, n}}{0} \mid \xi_{6}\right)+A_{2}\left(\frac{1}{k+1}\right)^{m_{s, r_{k}}+\bar{i}}\right. \\
& \left.\left.\beta_{s, r_{k}}^{-\bar{i}} G_{3}^{13}\left(\underset{0, m_{s, r_{k}}+\bar{i}, n}{0} \mid(k+1) \xi_{6}\right)\right)\right] \text {, }
\end{aligned}
$$

where $\xi_{5}=\frac{\beta_{s, r_{k}} \beta_{r_{k}, 2}}{\nu}$ and $\xi_{6}=\frac{\beta_{s, r_{k}} \beta_{r_{k}, 2}}{\bar{\nu}}$.

\section{Numerical and Simulation Results}

In this section, numerical and simulation results are presented to evaluate the impact of imperfect CSI/SIC, the number of relays, EH time fraction $(\alpha)$ and $\sigma_{e}^{2}$ on the considered NOMA system. Unless specified, the system parameter are as follows. The target data rate $r_{2}=r_{1}=0.5 \mathrm{bpcu}, \alpha=0.35$, energy conversion efficiency $\mu=0.9, m_{i, j}=2, \beta=0.15$, the channel gains $\lambda_{s, r_{k}}=\lambda_{r_{k}, 1}=1, \lambda_{r_{k}, 2}=0.5, \sigma_{e}^{2}=0.01, a_{1}=0.3$ and $M A X_{\text {particles }}=30$. [15]. The correctness of the derived analytical expressions is validated through Monte-Carlo simulations. Simulations are performed using Matlab, and analytical results are obtained using Mathematica. In the figures, (Sim.) denotes Matlab simulation result. In Fig. 2, the outage probability experienced by $U_{1}$ and $U_{2}$ under imperfect CSI/SIC is compared with perfect CSI/SIC for the considered NOMA system. Results show a significant impact on the outage probability of both users due to imperfect CSI/SIC. It is observed that at the outage of $10^{-1}$ with $\mathrm{K}=2, U_{1}$ with perfect CSI/SIC provides SNR gain of $5 \mathrm{~dB}$ over imperfect CSI/SIC case and $U_{2}$ with perfect CSI/SIC provides SNR gain of $10 \mathrm{~dB}$ over imperfect CSI/SIC. Whereas, at high SNR regime, the outage probability of users with imperfect CSI/SIC suffers outage floor and reaches a constant value of 0.038 and 0.12 for $U_{1}$ and $U_{2}$ respectively. With the increase in SNR, CEE increases and thus limits outage probability to further decrease and maintains a constant value for both users under imperfect CSI. However, in $U_{1}$, the residual interference due to imperfect SIC also increases with the increase in the SNR, thus causing degradation in the outage probability of $U_{1}$. Further, from the figure, a performance gain is observed as we increase the $\mathrm{K}$. A gain of $3 \mathrm{~dB}$ is observed for both users under perfect CSI/SIC, with the increase in relays from 2 to 5 for an outage probability of $10^{-2}$.

$$
\begin{aligned}
& I_{1}=e^{\hat{\beta}_{r_{k}, 1} \sigma_{e}^{2}} A_{1}\left(-\sigma_{e}^{2}\right)^{n-j}\left(\frac{1}{\zeta}\right)^{j}\left[\left(\frac{\hat{\beta}_{r_{k}, 1}}{\zeta}\right)^{-j} G_{3}^{1}{ }_{1}^{3}\left(\underset{0, m_{s, r_{k}}, j}{0} \mid \xi_{1}\right)+A_{2}\left(\frac{\hat{\beta}_{r_{k}, 1}}{\zeta}\right)^{-j}\left(\frac{1}{k+1}\right)^{m_{s, r_{k}}+\bar{i}} \hat{\beta}_{s, r_{k}}^{-\bar{i}}\right. \\
& \left.G_{3}^{1}{ }_{1}^{3}\left(\underset{0, m_{s, r_{k}}+\bar{i}, j}{0} \mid \xi_{1}(k+1)\right)\right]-e^{\xi_{3} \hat{\beta}_{r_{k}, 1}} A_{1}\left(-\xi_{3}\right)^{n-j}\left(\frac{1}{\bar{\zeta}}\right)^{j}\left[\left(\frac{\hat{\beta}_{r_{k}, 1}}{\bar{\zeta}}\right)^{-j} G_{3}^{1}{ }_{1}^{3}\left(\underset{0, m_{s, r_{k}}, j}{0} \mid \xi_{2}\right)+A_{2}\right. \\
& \left.\left(\frac{\hat{\beta}_{r_{k}, 1}}{\bar{\zeta}}\right)^{-j}\left(\frac{1}{k+1}\right)^{m_{s, r_{k}}+\bar{i}} \hat{\beta}_{s, r_{k}}^{-\bar{i}} G_{3}^{1}{ }_{1}^{3}\left(0, m_{s, r_{k}}+\bar{i}, j \mid \xi_{2}(k+1)\right)\right] \text {. } \\
& J_{1}=e^{\hat{\beta}_{r_{k}, 2} \sigma_{e}^{2}} A_{3}\left(-\sigma_{e}^{2}\right)^{n-j}\left(\frac{1}{\nu}\right)^{j}\left[\left(\frac{\hat{\beta}_{r_{k}, 2}}{\nu}\right)^{-j} G_{3}^{1}{ }_{1}^{3}\left(\underset{0, m_{s, r_{k}}, j}{0} \mid \xi_{5}\right)+A_{2}\left(\frac{\hat{\beta}_{r_{k}, 2}}{\nu}\right)^{-j}\left(\frac{1}{k+1}\right)^{m_{s, r_{k}}+\bar{i}} \hat{\beta}_{s, r_{k}}^{-\bar{i}}\right.
\end{aligned}
$$

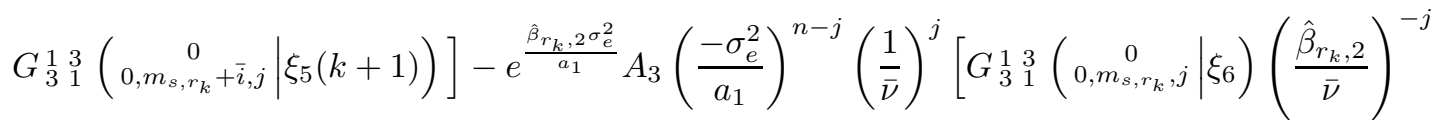

$$
\begin{aligned}
& \left.+A_{2}\left(\frac{\hat{\beta}_{r_{k}, 2}}{\bar{\nu}}\right)^{-j}\left(\frac{1}{k+1}\right)^{m_{s, r_{k}}+\bar{i}} \hat{\beta}_{s, r_{k}}^{-\bar{i}} G_{3}^{1} \frac{3}{1}\left(\begin{array}{c}
0, m_{s, r_{k}}+\bar{i}, j \\
0
\end{array} \xi_{6}(k+1)\right)\right] .
\end{aligned}
$$




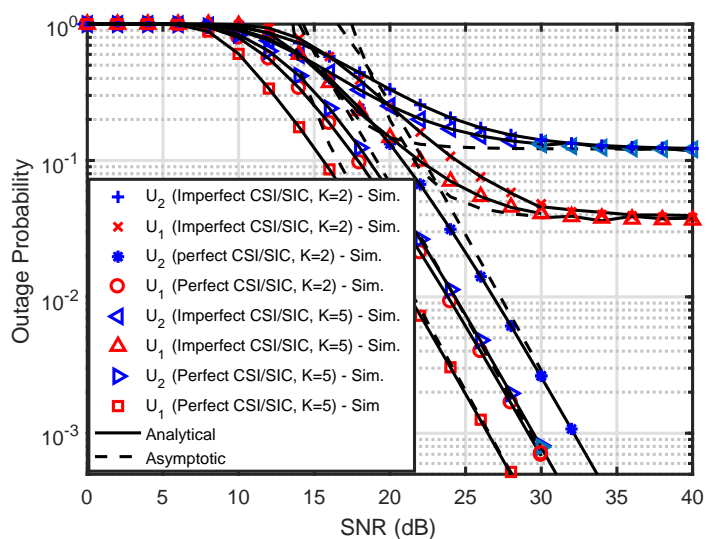

Fig. 2 Outage probability of $U_{1}$ and $U_{2}$ with respect to transmit SNR $\left(a_{2}=0.7, \alpha=0.35\right)$.

In the case of imperfect CSI/SIC, an SNR gain of $2 \mathrm{~dB}$ is observed at an outage probability of 0.1 and 0.2 for $U_{1}$ and $U_{2}$, respectively. Whereas at high value of SNR, due to the presence of CEE and residual interference due to imperfect SCI performance gain is not observed with the increase in K. It is also observed that the analytical results are perfectly matching with the simulation result. Further, the derived asymptotic outage results match the derived analytical and the simulation results at a high SNR value, which validates our results.

Fig. 3 investigates the impact of CEE $\sigma_{e}^{2}$ on $U_{1}$ and $U_{2}$ with the fixed residual interference due to SIC error $\beta$. It is observed the outage floor decreases with a decrease in $\sigma_{e}^{2}$ and approaches towards perfect CSI/SIC case for both the users. In $U_{1}$, for high CEE values $\sigma_{e}^{2}=0.01$ and $\sigma_{e}^{2}=0.005$, perfect CSI/SIC has an SNR gain of $4 \mathrm{~dB}$ and $3 \mathrm{~dB}$ for an outage of $10^{-1}$. However, for smaller values of CEE $\left(\sigma_{e}^{2}=0.001\right)$, the gain is $5 \mathrm{~dB}$ for an outage of $10^{-3}$. Thus, the outage probability is more limited by CEE than SIC error, as high CEE shows degradation in outage probability with the same value of $\beta$.

Fig. 4 and Fig. 5 illustrate the impact of $\alpha$, the fraction of EH time, on the outage probability of $U_{1}$ and $U_{2}$. In the given scenario, the $\alpha$ is ranged between 0.1 and 0.7 , whereas the $\rho$ and $\sigma_{e}^{2}$ are fixed at $20 \mathrm{~dB}$ and 0.01 respectively. In Fig. 4, the system's performance comparison under perfect CSI/SIC and imperfect $\mathrm{CSI} / \mathrm{SIC}$ are shown. With the increase in $\alpha$, the EH time increases, thus reducing the information processing time and hence outage probability of users increases for the entire time duration. The plot shows that the optimum value of $\alpha$, which minimizes the outage probability, lies in the range of 0.2 to 0.3 for both perfect and imperfect CSI/SIC. Further, it is observed that at a high value

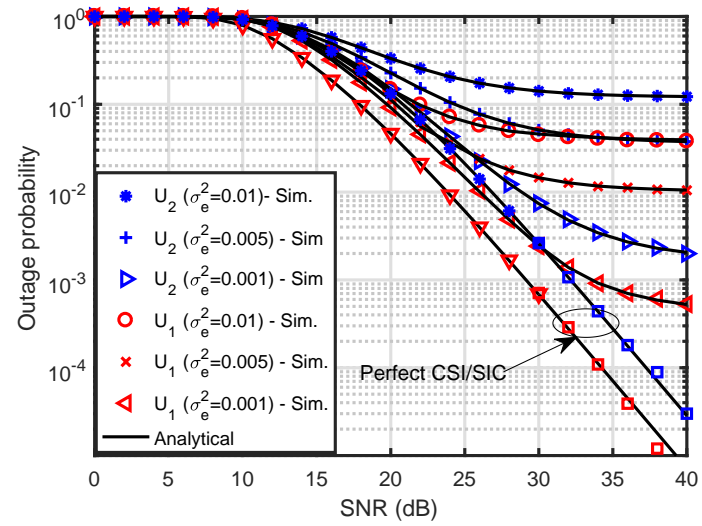

Fig. 3 Outage probability of $U_{1}$ and $U_{2}$ under imperfect CSI/SIC with respect to transmit SNR for different value of $\sigma_{e}^{2}\left(a_{2}=0.7, \alpha=0.35\right)$.

of $\alpha$, i.e., above 0.45 , the outage probability of users become one. With the increase in the $\alpha$, the threshold SNR, i.e., $\gamma_{t h 1}$ and $\gamma_{t h 2}$, also increases to maintain the constant target data rates $r_{1}$ and $r_{2}$, respectively. Thus, the outage probability criteria $\gamma_{t h 2}<\frac{a_{2}}{a_{1}}$ and $\gamma_{t h 1}<\frac{a_{1}}{\beta a_{2}}$ does not satisfied and makes the outage probability $P_{\text {out }, 1}=P_{\text {out }, 2}=1$. In Fig. 5, the impact of $a_{1}$ and $\beta$ on the outage probability of users under imperfect CSI/SIC is analyzed. In the case of $U_{2}$, it is observed that the outage probability with $a_{1}=0.1$ shows significant improvement over $a_{1}=0.3$ since more power is allocated to the symbol intended for $U_{2}$. However, in $U_{1}, P_{\text {out }, 1}$ tends to unity due to failure of outage criteria $\gamma_{t h 1}<\frac{a_{1}}{\beta a_{2}}$ and $\gamma_{t h 2}<\frac{a_{2}}{a_{1}}$. Considering the high residual interference due to imperfect SIC, i.e., $\beta=0.16$ and $\beta=0.1$, the outage criteria is not satisfied and leads to constant noise error floor. With a further decrease in residual interference i.e. $\beta=0.01$, the outage probability of $U_{1}$ reduces. Hence, selection of $a_{1}$ and $\beta$ plays a crucial role on the outage performance of $U_{1}$.

Fig. 6 shows the system throughput under optimized and non-optimized, i.e., (arbitrary) $\alpha=0.3$ value. It is observed that the system throughput of the optimized NOMA scheme under both perfect and imperfect SIC/CSI is enhanced compared to the non-optimized value. It is observed that at the system throughput of $0.3 \mathrm{bps} / \mathrm{Hz}$, the optimized value provides an SNR gain of $3 \mathrm{~dB}$ over the non-optimized fixed value. In Fig. 7 both users' ergodic rate under perfect CSI/SIC and imperfect CSI/SIC are plotted against SNR. It is observed from the figure that the ergodic rate of $U_{1}$ under perfect CSI/SIC outperforms the ergodic rate of all other cases. The ergodic rate of $U_{1}$ under perfect CSI/SIC increases linearly with the SNR, whereas in 


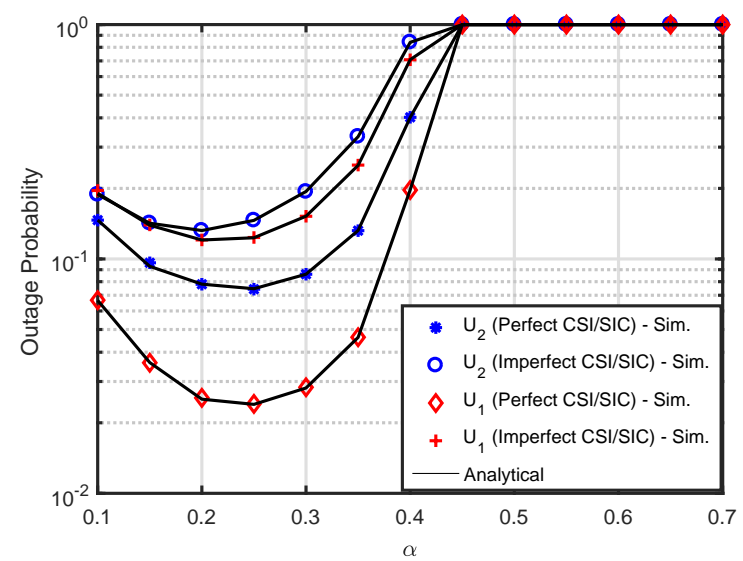

Fig. 4 Outage probability of $U_{1}$ and $U_{2}$ with respect to $\alpha(\mathrm{K}=2$, $\left.\rho=20 d B, a_{2}=0.7\right)$

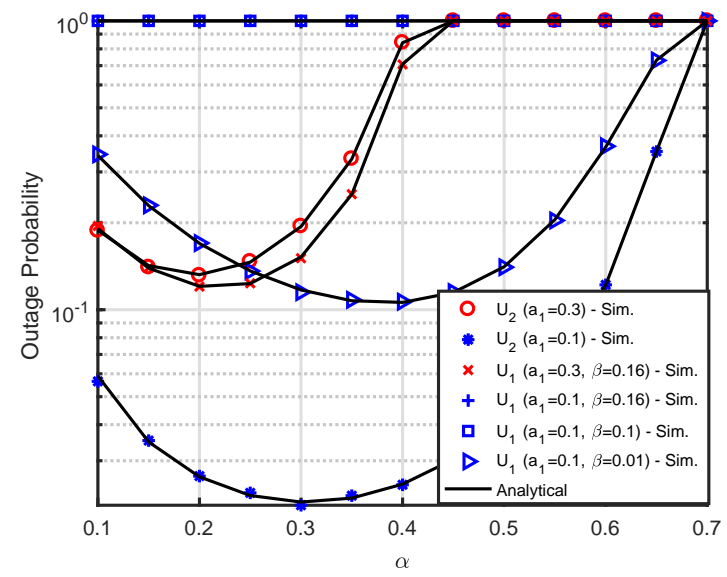

Fig. 5 Outage probability of $U_{1}$ and $U_{2}$ under imperfect CSI/SIC with respect to $\alpha\left(\mathrm{K}=2, \rho=20 d B, \sigma_{e}^{2}=0.01\right)$

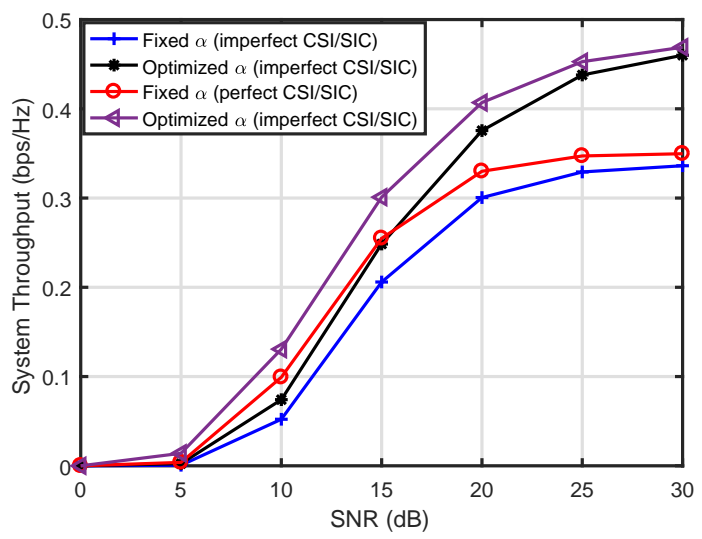

Fig. 6 System throughput with respect to transmit SNR, comparison study of optimized and non optimized fraction of EH time $\left(\mathrm{K}=2, a_{1}=0.3\right)$

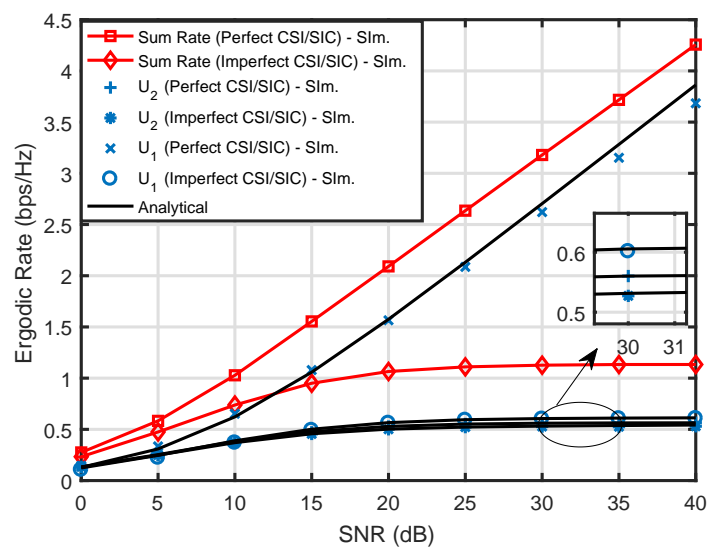

Fig. 7 Ergidic rate of $U_{1}$ and $U_{2}$ with respect to transmit SNR $\left(\mathrm{K}=2, a_{1}=0.3\right)$

case of $U_{2}$, the ergodic rate saturate at a high SNR region. The ergodic rate of $0.5 \mathrm{bps} / \mathrm{Hz}$ the $U_{1}$ provides an SNR gain of $7 \mathrm{~dB}$ over $U_{2}$ with perfect CSI/SIC. With the increase in SNR, the interference generated by the symbol of $U_{1}$ increases and leads to saturation in the ergodic rate plot. It is also observed that both users under imperfect CSI/SIC shows are marginal increasing trend at low SNR region and saturates at high SNR region. This is due to the increase in SNR, the interference due to imperfect CSI/SIC increases, thus the ergodic rate reduces effectively. Further, the NOMA system's sum rate (i.e., $r_{1}+r_{2}$ ) is also presented under perfect CSI/SIC and imperfect CSI/SIC. For the ergodic rate of $1 \mathrm{bps} / \mathrm{Hz}$, the perfect CSI/SIC case provides an SNR gain of $10 \mathrm{~dB}$ over imperfect CSI/SIC. It is observed that at high SNR values, there is a marginal gap between the analytical and simulated curve for $U_{1}$, which is because of the approximation used in the analytical expression.

\section{CONCLUSION}

In this paper, the analysis of downlink multiple EH relay-based NOMA system over Nakagami $-m$ fading is performed. The practical assumption of imperfect SIC and imperfect CSI were taken into consideration for investigation. The closed-form expressions for the outage probability, asymptotic outage probability, and ergodic rate of the users under imperfect CSI/SIC are obtained. The analytical results showed that the system under imperfect CSI/SIC provides massive performance degradation compared to the perfect CSI/SIC NOMA system. It is observed that the users' performance improved with increasing the total number of active relay nodes in the system. The significance of channel estimation error over the performance of users is analyzed. The impact of power allocation coefficient and a fraction of block time over the user performances are also analyzed. Further, the impact of SIC error over the performance of $U_{1}$ is 
analyzed. It is also observed that there is an optimum value of $\alpha$ for users' minimum outage probability. Obtained analytical results are validated through extensive simulation results. This generalized analysis is useful for future applications, including $\mathrm{UAV}, \mathrm{THz}$, and Internet of Things deployments for beyond 5G networks.

\section{APPENDIX A \\ PROOF OF LEMMA 1}

Proof. The closed-from expression of $(8)$ is derived as follows. Consider the outage probability definition given in (7). Substituting (2), (3), (4) and (5) in (7), on rearranging, we get

$$
\begin{aligned}
P_{\text {out }, 1}= & 1-\operatorname{Pr}\left\{\left|\hat{h}_{s, r}\right|^{2}>\frac{\gamma_{t h 2}\left(P_{s} \sigma_{e}^{2}+\sigma_{0}^{2}\right)}{\left(a_{2}-a_{1} \gamma_{t h 2}\right) P_{s}},\right. \\
& \left|\hat{h}_{r_{k}, 1}\right|^{2}>\frac{\gamma_{t h 2}\left(P_{r} \sigma_{e}^{2}+\sigma_{0}^{2}\right)}{\left(a_{2}-a_{1} \gamma_{t h 2}\right) P_{r}}, \\
& \left|\hat{h}_{s, r}\right|^{2}>\frac{\gamma_{t h 1}\left(P_{s} \sigma_{e}^{2}\left(a_{2}+\beta a_{1}\right)+\sigma_{0}^{2}\right)}{\left(a_{1}-\beta a_{2} \gamma_{t h 1}\right) P_{s}}, \\
& \left.\left|\hat{h}_{r_{k}, 1}\right|^{2}>\frac{\gamma_{t h 1}\left(P_{r} \sigma_{e}^{2}\left(a_{1}+\beta a_{2}\right)+\sigma_{0}^{2}\right)}{\left(a_{1}-\beta a_{2} \gamma_{t h 1}\right) P_{r}}\right\},
\end{aligned}
$$

According to $\mathrm{EH}$ criteria the transmitted power at relay is given as $P_{r}=\frac{2 P_{s} \mu\left|\hat{h}_{s, r}\right|^{2} \alpha}{1-\alpha}$. Substituting $P_{r}$ in A.1], and after some simplification A.11 is given as

$$
\begin{aligned}
P_{\text {out }, 1}= & 1-\operatorname{Pr}\left\{\left|\hat{h}_{s, r}\right|^{2}>\psi,\left|h_{r_{k}, 1}\right|^{2}>\frac{\lambda_{1}}{\left|\hat{h}_{s, r}\right|^{2}}\right. \\
& \left.+\Omega_{1},\left|h_{r_{k}, 1}\right|^{2}>\frac{\lambda_{2}}{\left|\hat{h}_{s, r}\right|^{2}}+\Omega_{2}\right\}, \quad \text { (A }
\end{aligned}
$$

where, $\Delta_{1}=\frac{\gamma_{t h 2}\left(\sigma_{e}^{2}+(1 / \rho)\right)}{\left(a_{2}-a_{1} \gamma_{t h 2}\right)}, \lambda_{1}=\frac{\gamma_{t h 2}(1-\alpha)}{\left(a_{2}-a_{1} \gamma_{t h 2}\right) \alpha \mu 2 \rho}$, $\lambda_{2}=\frac{\gamma_{t h 1}(1-\alpha)}{\left(a_{1}-a_{2} \beta \gamma_{t h 1}\right) \alpha \mu 2 \rho}, \Omega_{1}=\frac{\gamma_{t h 2} \sigma_{e}^{2}}{a_{2}-a_{1} \gamma_{t h 2}}, \Omega_{2}=$ $\frac{\gamma_{t h 2}\left(a_{1}+a_{2} \beta\right) \sigma_{e}^{2}}{a_{1}-a_{2} \beta \gamma_{t h 2}}, \psi=\max \left[\Delta_{1}, \frac{\gamma_{t h 1}\left(P_{s} \sigma_{e}^{2}\left(a_{1}+a_{2} \beta\right)+\sigma_{0}^{2}\right)}{\left(a_{1}-a_{2} \beta \gamma_{t h 1}\right) P_{s}}\right]$.

$$
\begin{aligned}
P_{\text {out }, 1}= & 1-\left[\operatorname { P r } \left\{\left|\hat{h}_{s, r}\right|^{2}>\psi,\left|h_{r_{k}, 1}\right|^{2}>\frac{\lambda_{1}}{\left|\hat{h}_{s, r}\right|^{2}}\right.\right. \\
& \left.+\Omega_{1},\left|\hat{h}_{s, r}\right|^{2}>\frac{\lambda_{2}-\lambda_{1}}{\Omega_{1}-\Omega_{2}}\right\} \\
& +\operatorname{Pr}\left\{\left|\hat{h}_{s, r}\right|^{2}>\psi,\left|h_{r_{k}, 1}\right|^{2}>\frac{\lambda_{2}}{\left|\hat{h}_{s, r}\right|^{2}}\right. \\
& \left.\left.+\Omega_{2},\left|\hat{h}_{s, r}\right|^{2}<\frac{\lambda_{2}-\lambda_{1}}{\Omega_{1}-\Omega_{2}}\right\}\right] .
\end{aligned}
$$

Let $P_{\text {out }, 1}=1-(A+B)$, where $A$ and $B$ are separately evaluated as follows. Assume the links in the network to be independent, $\gamma_{t h 1}<\frac{a_{1}}{a_{2} \beta}, \gamma_{t h 2}<\frac{a_{2}}{a_{1}}, \lambda_{2}>\lambda_{1}$ and $\Omega_{1}>\Omega_{2}$. Since i.i.d Nakagami fading is assumed, the channel power gains $\left|\hat{h}_{i, j}\right|$ are gamma function. The
PDF and CDF [36] of $\left|\hat{h}_{i, j}\right|^{2}$ with parameter $m_{i, j}$ and $\hat{\lambda}_{i, j}$ is given as

$$
\begin{gathered}
f_{\left|\hat{h}_{i, j}\right|^{2}}(x)=\left(\frac{m_{i, j}}{\hat{\lambda}_{i, j}}\right)^{m_{i, j}} \frac{x^{m_{i, j}-1}}{\Gamma\left(m_{i, j}\right)} e^{\frac{-x m_{i, j}}{\hat{\lambda}_{i, j}}}, \\
F_{\left|\hat{h}_{i, j}\right|^{2}}(x)=1-e^{\frac{-x m_{i, j}}{\hat{\lambda}_{i, j}}} \sum_{n=0}^{m_{i, j}-1} \frac{1}{n !}\left(\frac{x m_{i, j}}{\hat{\lambda}_{i, j}}\right)^{n},
\end{gathered}
$$

Let $\hat{\beta}_{i, j}=\frac{m_{i, j}}{\hat{\lambda}_{i, j}}$, since PRS with K-relays is performed,the CDF and PDF of the $\left|\hat{h}_{s, r}\right|^{2}$ is given by

$$
\begin{aligned}
F_{\left|\hat{h}_{s, r}\right|^{2}}(x)= & {\left[1-e^{-x \hat{\beta}_{s, r_{k}}} \sum_{n=0}^{m_{s, r_{k}}-1} \frac{1}{n !}\left(x \hat{\beta}_{s, r_{k}}\right)^{n}\right]_{(\text {A.6) }}^{K}, } \\
f_{\left|\hat{h}_{s, r}\right|^{2}}(x)= & K \hat{\beta}_{s, r_{k} m_{k}}^{m_{s, r_{k}}} \frac{x^{m_{s, r_{k}}-1}}{\left(m_{s, r_{k}}-1\right) !} e^{-x \hat{\beta}_{s, r_{k}}}\left[1+\sum_{k=1}^{K-1}\right. \\
& (-1)^{k}\left(\begin{array}{c}
K-1 \\
k
\end{array}\right) e^{-k x \hat{\beta}_{s, r_{k}}} \\
& \left.\left(\sum_{n=0}^{m_{s, r_{k}}-1} \frac{1}{n !}\left(x \hat{\beta}_{s, r_{k}}\right)^{n}\right)^{k}\right],
\end{aligned}
$$

By using the Binomial expression for the term $\left(\sum_{n=0}^{m_{s, r_{k}}-1} \frac{1}{n !}\left(x \hat{\beta}_{s, r_{k}}\right)^{n}\right)^{k}$, the Binomial expansion is given as [18]

$$
\begin{aligned}
& =\sum_{i_{1}=0}^{k} \sum_{i_{2}=0}^{k-i_{2}} \ldots \sum_{i_{m_{s, r_{k}}-1}=0}^{k-i_{1} \cdots i_{m_{s, r_{k}}-2}}\left(\begin{array}{c}
k \\
i_{1}
\end{array}\right)\left(\begin{array}{c}
k-i_{1} \\
i_{2}
\end{array}\right) \ldots \\
& \left(\begin{array}{c}
k-i_{1}-\ldots i_{m_{s, r_{k}}}-2 \\
i_{m_{s, r_{k}}-1}
\end{array}\right) \prod_{r=0}^{m_{s, r_{k}}-2}\left(\frac{\left(\hat{\beta}_{s, r_{k}} x\right)^{r}}{r !}\right)^{i_{r+1}} \\
& \left(\frac{\hat{\beta}_{s, r_{k}}^{m_{s, r_{k}}-1} x}{\left(m_{s, r_{k}}-1\right) !}\right)^{k-i_{1}-\ldots i_{m_{s}, r_{k}-1}}
\end{aligned}
$$

Substituting A.8 in A.7), the CDF is obtained. By utilizing the above equation $\mathrm{A}$ is obtained as follows

$$
\begin{aligned}
& A=\int_{x=\gamma}^{\infty}\left[1-F_{r_{k}, 1}\left(\frac{\lambda_{1}}{x}+\Omega_{1}\right)\right] f_{s, r_{k}}(x) d x, \\
& A=\int_{x=\gamma}^{\infty} e^{-\hat{\beta}_{r_{k}, 1}\left(\frac{\lambda_{1}}{x}+\Omega_{1}\right)} \sum_{n=0}^{m_{r_{k}, 1}-1} \frac{K}{n !} \hat{\beta}_{s, r_{k}}^{m_{s, r_{k}}} e^{-x \hat{\beta}_{s, r_{k}}} \\
& \left(\hat{\beta}_{r_{k}, 1}\left(\frac{\lambda_{1}}{x}+\Omega_{1}\right)\right)^{n} \frac{x^{m_{s, r_{k}}-1}}{\left(m_{s, r_{k}}-1\right) !}\left[1+\sum_{k=1}^{K-1}\right. \\
& \left.(-1)^{k}\left(\begin{array}{c}
K-1 \\
k
\end{array}\right) e^{-k x \hat{\beta}_{s, r_{k}}} \bigcup_{k} \Xi_{1, k} \Xi_{2, k} x^{\bar{i}}\right] d x,
\end{aligned}
$$


where $\gamma=\max \left[\psi,\left(\frac{\lambda_{2}-\lambda_{1}}{\Omega_{1}-\Omega_{2}}\right)\right], \bigcup_{k}=$

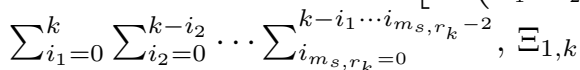
$\left(\begin{array}{c}k \\ i_{1}\end{array}\right)\left(\begin{array}{c}k-i_{1} \\ i_{2}\end{array}\right) \ldots\left(\begin{array}{c}k-i_{1}-\ldots i_{m_{s}, r_{k}-2} \\ i_{m_{s, r_{k}}-1}\end{array}\right), \Xi_{2, k}$ $\prod_{r=0}^{m_{s, r_{k}}-2}\left(\frac{\hat{\beta}_{s, r_{k}}^{r}}{r !}\right)^{i_{r+1}}\left(\frac{\hat{\beta}_{s, r_{k}}^{m_{s, r_{k}}-1}}{\left(m_{s, r_{k}}-1\right) !}\right)^{k-i_{1}-\ldots i_{m_{s}, r_{k}}-1}$ and $\bar{i}=\left(m_{s, r_{k}}-1\right)\left(k-i_{1}\right)-\left(m_{s, r_{k}}-2\right) i_{2}-\left(m_{s, r_{k}}-\right.$ 3) $i_{3}-\ldots i_{m_{s, r_{k}}-1}$. Using Binomial expansion on $\left(\frac{\lambda_{1}}{x}+\Omega_{1}\right)^{n}$, the above equation can further simplified as

$$
\begin{aligned}
A= & e^{-\hat{\beta}_{r_{k}, 1} \Omega_{1}} \sum_{n=0}^{m_{r_{k}, 1}-1} \frac{1}{n !} \hat{\beta}_{r_{k}, 1}^{n} K \hat{\beta}_{s, r_{k}}^{m_{s, r_{k}}} \frac{1}{\left(m_{s, r_{k}}-1\right) !} \\
& \sum_{j=0}^{n}\left(\begin{array}{c}
n \\
j
\end{array}\right) \lambda_{1}^{j} \Omega_{1}^{n-j}\left[\int_{x=\gamma}^{\infty} x^{m_{s, r_{k}}-j-1} e^{-x \hat{\beta}_{s, r_{k}}}\right. \\
& e^{\frac{-\hat{\beta}_{r_{k}, 1} \lambda_{1}}{x}} d x+\sum_{k=1}^{K-1}(-1)^{k}\left(\begin{array}{c}
K-1 \\
k
\end{array}\right) \bigcup_{k} \Xi_{1, k} \Xi_{2, k} \\
& \left.\int_{x=\gamma}^{\infty} e^{-x(k+1) \hat{\beta}_{s, r_{k}}} x^{m_{s, r_{k}}-j+\bar{i}-1} e^{\frac{-\hat{\beta}_{r_{k}, 1} \lambda_{1}}{x}} d x\right] .
\end{aligned}
$$

Now, using Taylor's series expansion, as $e^{\frac{-\hat{\beta}_{r_{k}, 1} \lambda_{1}}{x}}=$ $\sum_{l_{1}=0}^{N_{t}} \frac{(-1)^{l_{1}}}{l_{1} !}\left(\frac{\hat{\beta}_{r_{k}, 1} \lambda_{1}}{x}\right)^{l_{1}}$ where $N_{t} \in\{2,3, \ldots \infty\}$ and using [35, eq. (3.351.4)] the A.11 can be simplified. Further, the B is simplified as follows

$$
B=\int_{x=\psi}^{\frac{\lambda_{2}=\lambda_{1}}{\Omega_{1}-\Omega_{2}}}\left[1-F_{r_{k}, 1}\left(\frac{\lambda_{2}}{x}+\Omega_{2}\right)\right] f_{s, r}(x) d x .
$$

The (A.12), can be simplified by following the same approach as used in A.9, the obtained result $P_{\text {out }, 1}=$ $1-(A+B)$ is given by (8).

\section{APPENDIX B}

\section{PROOF OF LEMMA 2}

Proof. The closed form expression of (10) is obtained as follows. Substitute (2) and (6) in (9), after some manipulation (9) can be simplified as

$$
\begin{aligned}
P_{\text {out }, 2}= & 1-\operatorname{Pr}\left\{\left|\hat{h}_{s, r}\right|^{2}>\frac{\gamma_{t h 2}\left(P_{s} \sigma_{e}^{2}+\sigma_{0}^{2}\right)}{\left(a_{2}-a_{1} \gamma_{t h 2}\right) P_{s}},\right. \\
& \left.\left|\hat{h}_{r_{k}, 2}\right|^{2}>\frac{\gamma_{t h 2}\left(P_{r} \sigma_{e}^{2}+\sigma_{0}^{2}\right)}{\left(a_{2}-a_{1} \gamma_{t h 2}\right) P_{r}}\right\},
\end{aligned}
$$

Substituting $P_{r}=\frac{2 P_{s} \alpha \mu\left|\hat{h}_{s, r}\right|^{2}}{1-\alpha}$, B.1 can be further reduced as

$$
\begin{aligned}
P_{\text {out }, 2}= & 1-\operatorname{Pr}\left\{\left|\hat{h}_{s, r}\right|^{2}>\Delta_{1},\right. \\
& \left.\left|\hat{h}_{r_{k}, 1}\right|^{2}>\frac{\lambda_{1}}{\left|\hat{h}_{s, r}\right|^{2}}+\Omega_{1}\right\},
\end{aligned}
$$

$P_{\text {out }, 2}=1-\int_{x=\Delta_{1}}^{\infty}\left[1-F_{r_{k}, 2}\left(\frac{\lambda_{1}}{x}+\Omega_{1}\right)\right] f_{s, r}(x) d x$

The integration in B.3 can be simply simplify by following the same approach as of A.9 of Appendix A. The obtained closed-form expression is given by (10).

\section{APPENDIX C \\ PROOF OF LEMMA 3}

Proof. Considering the perfect CSI and perfect SIC condition on the SINR's equation (2), (3), (4) and (5). The outage probability of $U_{1}$ under perfect SIC and perfect CSI is given as

$$
\begin{aligned}
P_{\text {out }, 1}^{P}= & 1-\operatorname{Pr}\left\{\frac{\left|h_{s, r}\right|^{2} P_{s} a_{2}}{\left|h_{s, r}\right|^{2} P_{s} a_{1}+\sigma_{0}^{2}} \cdot>\gamma_{t h 2},\right. \\
& \frac{\left|h_{r_{k}, 1}\right|^{2} P_{r} a_{2}}{\left|h_{r_{k}, 1}\right|^{2} P_{r} a_{1}+\sigma_{0}^{2}}>\gamma_{t h 2}, \frac{\left|h_{s, r}\right|^{2} P_{s} a_{1}}{\sigma_{0}^{2}} \\
& \left.>\gamma_{t h 1}, \frac{\left|h_{r_{k}, 1}\right|^{2} P_{r} a_{1}}{\sigma_{0}^{2}} \cdot>\gamma_{t h 1}\right\} .
\end{aligned}
$$

substituting $P_{r}=\frac{2 P_{s} \mu\left|h_{s, r}\right|^{2} \alpha}{1-\alpha}$ and after some manipulation, C.1 further simplified as

$$
P_{\text {out }, 1}^{P}=1-\operatorname{Pr}\left\{\left|h_{s, r}\right|^{2}>\Delta_{3},\left|h_{r_{k}, 1}\right|>\frac{\Delta_{2}}{\left|h_{s, r}\right|^{2}}\right\},
$$

where $\Delta_{3}=\max \left[\frac{\gamma_{t h 2} \sigma_{0}^{2}}{\left(a_{2}-a_{1} \gamma_{t h 2}\right) P_{s}}, \frac{\gamma_{t h 1} \sigma_{0}^{2}}{a_{1} P_{s}}\right], \quad \Delta_{2}=$ $\max \left[\lambda_{1}, \frac{\gamma_{t h 1}(1-\alpha)}{a_{1} \alpha 2 \mu P_{s}}\right]$. Let $P_{o u t, 1}^{P}=1-D$, where $\mathrm{D}$ is evaluated as follow

$$
\begin{aligned}
D= & \int_{x=\Delta_{3}}^{\infty}\left[1-F_{r_{k}, 1}\left(\frac{\Delta_{2}}{x}\right)\right] f_{s, r_{k}}(x) d x, \quad \text { (C.3) } \\
= & \sum_{n=0}^{m_{r_{k}, 1}-1} \frac{1}{\left(m_{s, r_{k}}-1\right) !}\left(\frac{m_{r_{k}, 1} \Delta_{2}}{\lambda_{r_{k}, 1}}\right)^{n}\left(\frac{m_{s, r_{k}}}{\lambda_{s, r_{k}}}\right)^{m_{s, r_{k}}} \\
& \frac{K}{n !}\left[\int_{x=\Delta_{3}}^{\infty} x^{m_{s, r_{k}}-n-1} e^{\frac{m_{s, r_{k}} x}{\lambda_{s, r_{k}}}} e^{\frac{-m_{r_{k}, 1} \Delta_{2}}{\lambda_{r_{k}, 1^{x}}}} d x\right. \\
& +\sum_{k=1}^{K-1}(-1)^{k}\left(\begin{array}{c}
K-1 \\
k
\end{array}\right) \bigcup_{k} \Xi_{1, k} \Xi_{2, k} \int_{x=\Delta_{3}}^{\infty} \\
& \left.e^{\frac{-x(k+1) m_{s, r_{k}}}{\lambda_{s, r_{k}}}} x^{m_{s, r_{k}}-n+\bar{i}-1} e^{\frac{-m_{r_{k}, 1} \Delta_{2}}{\lambda_{r_{k}, 1^{x}}}} d x\right]
\end{aligned}
$$

The integration in (??), is same as in (A.11) in appendix A. Hence, D can be obtained following the similar procedure as adopted in A.11, which is given by (11). 


\section{REFERENCES}

[1] Z. Ding, X. Lei, G. K. Karagiannidis, R. Schober, J. Yuan, and V. K. Bhargava, "A survey on non-orthogonal multiple access for 5G networks: Research challenges and future trends," IEEE J. Sel. Areas Commun., vol. 35, no. 10, pp. 2181-2195, Jul. 2017.

[2] P. Swami, V. Bhatia, S. Vuppala, and T. Ratnarajah, "A cooperation scheme for user fairness and performance enhancement in NOMA-HCN," IEEE Trans. Veh. Technol., vol. 67, no. 12, pp. 11965-11978, Oct. 2018

[3] Y. Liu, Z. Qin, M. Elkashlan, Z. Ding, A. Nallanathan, and L. Hanzo, "Nonorthogonal Multiple Access for $5 \mathrm{G}$ and Beyond," IEEE Proc., vol. 105, no. 12, pp. 2347-2381, Dec. 2017.

[4] J. Kim and I. Lee, "Non-Orthogonal Multiple Access in Coordinated Direct and Relay Transmission," IEEE Commun. Lett., vol. 19, no. 11, pp. 2037-2040, Nov. 2015.

[5] Z. Ding, M. Peng, and H. V. Poor, "Cooperative non-orthogonal multiple access in 5G systems," IEEE Commun. Lett., vol. 19, no. 8, pp. 1462-1465, Jun. 2015.

[6] J. Men, J. Ge, and C. Zhang, "Performance analysis of nonorthogonal multiple access for relaying networks over Nakagami-m fading channels," IEEE Trans. Veh. Technol., vol. 66, no. 2, pp. 1200-1208, Feb. 2017.

[7] Z. Ding, H. Dai, and H. V. Poor, "Relay selection for cooperative NOMA," IEEE Wireless Commun. Lett., vol. 5, no. 4, pp. 416419, Aug. 2016.

[8] P. Xu, Z. Yang, Z. Ding, and Z. Zhang, "Optimal relay selection schemes for cooperative NOMA," IEEE Trans. on Veh. Technol., vol. 67, no. 8, pp. 7851-7855, Aug. 2018.

[9] Y. Li, Y. Li, X. Chu, Y. Ye, and H. Zhang, "Performance analysis of relay selection in cooperative NOMA networks," IEEE Commun. Lett., vol. 23, no. 4, pp. 760-763, Apr. 2019.

[10] S. Lee, D. B. da Costa, Q. Vien, T. Q. Duong, and R. T. de Sousa, "Non-orthogonal multiple access schemes with partial relay selection," IET Commun., vol. 11, no. 6, pp. 846-854, Apr. 2017.

[11] J. Jose, P. Shaik, and V. Bhatia, "VFD-NOMA under imperfect SIC and residual inter-relay interference over generalized nakagami-m fading channels," IEEE Commun. Lett., pp. 1-1, 2020.

[12] Y. Liu, Z. Ding, M. Elkashlan, and H. V. Poor, "Cooperative nonorthogonal multiple access with simultaneous wireless information and power transfer," IEEE J. Sel. Areas Commun., vol. 34 no. 4, pp. 938-953, Apr. 2016.

[13] H. Dac-Binh and N. Sang Quang, "Outage performance of energy harvesting DF relaying NOMA networks," Mobile Netw. and Appl., vol. 23, no. 6, pp. 1572-1585, Dec. 2018.

[14] Y. Zhang and J. Ge, "Performance analysis for non-orthogonal multiple access in energy harvesting relaying networks," IET Commun., vol. 11, no. 11, pp. 1768-1774, Aug. 2017.

[15] H. Tran Manh, N. Tan. Nguyen Trungand Hoang, and P. Hiep, "Performance analysis of decode-and-forward partial relay selection in NOMA systems with RF energy harvesting," Wireless Netw., vol. 25, no. 8, pp. 4585-4595, Feb. 2019.

[16] X. Li, J. Li, P. T. Mathiopoulos, D. Zhang, L. Li, and J. Jin, "Joint impact of hardware impairments and imperfect CSI on cooperative SWIPT NOMA multi-relaying systems," Proc. IEEE/CIC Int. Conf. commun. China, pp. 95-99, Jul. 2018.

[17] D. Do, M. Vaezi, and T. Nguyen, "Wireless powered cooperative relaying using NOMA with imperfect CSI," in Proc. IEEE Globecom Workshops, Dec. 2018, pp. 1-6.

[18] J. Men, J. Ge, and C. Zhang, "Performance analysis for downlink relaying aided non-orthogonal multiple access networks with imperfect CSI over Nakagami- $m$ fading," IEEE Access, vol. 5, pp. 998-1004, Mar. 2017.

[19] P. Shaik, P. K. Singya, and V. Bhatia, "On impact of imperfect CSI over hexagonal QAM for TAS/MRC-MIMO cooperative relay network," IEEE Commun. Lett., vol. 23, no. 10, pp. 17211724, Oct. 2019.

[20] E. N. Papasotiriou, A. A. Boulogeorgos, and A. Alexiou, "Performance analysis of $\mathrm{THz}$ wireless systems in the presence of antenna misalignment and phase noise," IEEE Commun. Lett. vol. 24, no. 6, pp. 1211-1215, Jun. 2020.

[21] M. Alzenad and H. Yanikomeroglu, "Coverage and rate analysis for unmanned aerial vehicle base stations with LoS/NLoS propagation," in Proc. IEEE Globecom Commun. Conf. Workshops, Dec. 2018, pp. 1-7.

[22] S. M. Kay, Fundamental of statistical signal processing: estimation theory. Prentice-hall, 1993.

[23] X. Liang, Y. Wu, D. W. K. Ng, S. Jin, Y. Yao, and T. Hong, "Outage probability of cooperative NOMA networks under imperfect CSI with user selection," IEEE Access, vol. 8, pp. 117921117931 , May 2020

[24] D. Do, T. Anh Le, T. N. Nguyen, X. Li, and K. M. Rabie, "Joint impacts of imperfect CSI and imperfect SIC in cognitive radioassisted NOMA-V2X communications," IEEE Access, vol. 8, pp. $128629-128645$, Jul. 2020.

[25] Taesang Yoo and A. Goldsmith, "Capacity and power allocation for fading MIMO channels with channel estimation error," IEEE Trans. on Inf. Theory, vol. 52, no. 5, pp. 2203-2214, May 2006.

[26] F. Fang, H. Zhang, J. Cheng, S. Roy, and V. C. M. Leung, "Joint user scheduling and power allocation optimization for energyefficient noma systems with imperfect CSI," IEEE J. Sel. Areas in Commun., vol. 35, no. 12, pp. 2874-2885, Dec. 2017.

[27] L. L. Scharf, Statistical Signal Processing: Detection, Estimation, and Time-series Analysis. Reading, MA: Addison-Wesley, 1991.

[28] I. Krikidis, J. Thompson, S. Mclaughlin, and N. Goertz, "Amplify-and-forward with partial relay selection," IEEE Commun. Lett., vol. 12, no. 4, pp. 235-237, Apr. 2008.

[29] S. Parvez, D. Kumar, and V. Bhatia, "On performance of SWIPT enabled two-way relay system with non-linear power amplifier," in National Conf. on Commun., Feb. 2020, pp. 1-6.

[30] S. Parvez, P. K. Singya, and V. Bhatia, "On ASER analysis of energy efficient modulation schemes for a device-to-device MIMO relay network," IEEE Access, vol. 8, pp. 2499-2512, Jan. 2020.

[31] P. Shaik, P. K. Singya, N. Kumar, K. K. Garg, and V. Bhatia, "On impact of imperfect CSI over SWIPT device-to-device (D2D) MIMO relay systems," in 2020 Int. Conf. on Signal Process. and Commun., 2020, pp. 1-5.

[32] W. Shuming, Z. Yudong, W. Shuihua, and J. Genlin, "A comprehensive survey on particle swarm optimization algorithm and its applications," Math. Problems Eng., pp. 1211-1215, Feb. 2015.

[33] M. Song and M. Zheng, "Energy efficiency optimization for wireless powered sensor networks with nonorthogonal multiple access," IEEE Sensors Lett., vol. 2, no. 1, pp. 1-4, Mar. 2018.

[34] A. Masaracchia, D. B. Da Costa, T. Q. Duong, M. Nguyen, and M. T. Nguyen, "A PSO-based approach for user-pairing schemes in NOMA systems: Theory and applications," IEEE Access, vol. 7, pp. 90 550-90 564, Jul. 2019.

[35] I. S. Gradshteyn and I. M. Ryzhik, Table of integrals, series, and products. Academic Press, 2014.

[36] A. Goldsmith, Wireless Communications. Cambridge University Press, 2005. 